'Division of Infection, Immunity and Respiratory Medicine, University of Manchester, Manchester, UK. ${ }^{2}$ Allergy Unit and Allergy Research Laboratory, Regional University Hospital of Malaga and Biomedical Research Institute of Malaga (IBIMA), Malaga, Spain. ${ }^{3}$ Priority Area Asthma and Allergy, Leibniz Center for Medicine and Biosciences, Research Center Borstel, Borstel, Germany. ${ }^{4}$ Airway Research Center North, member of the German Center for Lung Research (DZL), Borstel, Germany. ${ }^{5}$ Division of Asthma, Allergy and Lung Biology, Department of Paediatric Allergy, Children's Allergy Service, King's College London, and Guy's and St Thomas' National Health Service Foundation Trust, London, UK. ${ }^{6}$ All authors contributed equally.

\title{
ERS Early Career Members meet EAACI Junior Members: the launch of a strong, fruitful collaboration
}

\section{Early Career Forum}

Training, development and support in the career progression of junior members are of utmost importance and represent high priorities for both the European Respiratory Society (ERS) and the European Academy of Allergy and Clinical Immunology (EAACI). Apart from clinical and research fellowships, and educational events, both societies have invested in their respective juniors' boards. These aim to highlight the needs of the juniors, organise educational activities targeted to young clinicians and scientists, and promote active participation of early career members in the respective society's activities. Both ERS Early Career Members Committee (ECMC) and EAACl Junior Members' Assembly (JMA) board have been very active over past years, which has led numerous initiatives.

Respiratory medicine and allergology are interlinked specialties and we therefore considered that there are common grounds for collaboration between the ERS ECMC and EAACI JMA boards. Our aims are to promote educational and scientific activities in overlapping areas of interest, as well as to exchange ideas and experiences on the organisation of the juniors' activities, and develop a professional network. Several discussions taking place in a very friendly environment at the annual congresses of both societies led to the implementation of our initial joint steps. Here, we introduce our groups and future plans for our collaboration.

\section{The ERS Early Career Member Committee}

Every ERS member below the age of 40 years is termed an "Early Career Member". These comprise about 30\% ( 9000$)$ of all ERS members. In order to represent the special needs and wishes of all Early Career Members within the society, the ERS founded the ECMC at its International Congress in Vienna in 2012. The ECMC is comprised of 11 members, each representing one of the 11 scientific assemblies of the ERS. Representatives are elected by the members of the respective assemblies. The current composition and chair of the ECMC can be found at https://www.ersnet.org/the-society/who-we-are/ leadership/early-career-members-committee.

This committee mainly aims to promote the mission of the ERS, while providing support and resources for the professional development of Early Career Members. Along this line, ECMC members have a presence in the ERS Executive Committee, Science Council, Education Council, ERS publications and HERMES (Harmonised Education in Respiratory
Cite as: Mathioudakis AG, Eguiluz Gracia I, Bartel S, et al. ERS Early Career Members meet EAACI Junior Members: the launch of a strong, fruitful collaboration. Breathe 2017; 13: $231-233$. 
Medicine for Respiratory Specialists), as well as EBAP (European Board for Accreditation in Pneumology) and ELF (European Lung Foundation). Further, the ECMC organises sessions specially dedicated to professional and career development at every International Congress, and every Lung Science Conference in Estoril, Portugal. To enable and encourage Early Career Members to get in contact with members of the ECMC, and thus become actively involved in the ERS, the ECMC sessions are regularly followed by a networking evening/ cocktail.

\section{The EAACI Junior Members' Assembly Board}

The EAACI JMA is the largest EAACI Section, with approximately 4300 clinicians and scientists under the age of 36 years working in the field of allergy and clinical immunology. EAACI JMAs benefit from free EAACl membership and reduced registration fees for all EAACl events. In addition, they enjoy free online access to the $E A A C l$ journals and educational resources, and the opportunity to apply for the EAACI Mentorship Programme, Clinical and Research Fellowships, Scholarships for the Annual Congress and travel grants for the Focused Meetings and Allergy Schools. The JMA board (formerly known as the JM Working Group), was founded in 2001 [1]. It is the representative body of the JMA within EAACl as a structure and consists of a Chair, Secretary, Past Chair, and one Representative from each of the five EAACI Sections and the six EAACI Interest Groups. The JMA board Chair is a voting member of the EAACI Executive Committee and participates in other EAACl committees too.

The JMA board coordinates a number of initiatives, some of which are:

- definition and operation of the Junior Memberdedicated sessions during the EAACI Annual Congress

- Junior Member social media accounts (on Facebook, Twitter and Linkedln) aiming to provide a modern platform of communication and networking for the juniors

- Junior Member-dedicated webpages on eaaci. org where web visitors can learn more about the JMA board as well as the latest JMA news

- collaboration with the editorial boards of the three EAACl journals, Allergy, Paediatric Allergy and Immunology and Clinical and Translational Allergy; these collaborations aim to increase the visibility of the articles published in these journals mainly by means of social media posts and webcasted interviews [2]

- the Mentorship Programme, which supports communication between seniors and juniors

- translations from English into other main European languages of EAACI Position Papers, International Consensus documents [3,4] and webpages on EAACl's campaigns (e.g. bewareofallergy.com)

\section{Outline of the collaboration between the ERS ECMC and EAACI JMA}

The collaboration between the ERS ECMC and EAACI JMA Board intends to increase the opportunities for scientific and professional development of the young members of both organisations, with a special emphasis on the areas of overlapping scientific interest such as asthma, allergy and lung immunology. The reciprocal funding provided by both organisations for junior board representatives to attend the other society's annual meeting facilitates the interaction between the two junior boards. The EAACI Annual Congress already offers dedicated sessions within its scientific programme where the young members of both organisations have the opportunity to share their recent research findings with the rest of the delegates and the ERS ECMC is now working towards the establishment of such sessions during the ERS International Congress. The friendly atmosphere of these sessions facilitates the exchange of scientific ideas and the open-minded communication between the speakers and the audience.

Along these lines, one of the first initiatives of our collaboration was the development of a Task Force proposal, organised and led by $A$. Mathioudakis (ERS ECMC Assembly 5 representative) and O. Tsilochristou (EAACI JMA Board Chair), which was submitted to, approved and cofinanced by both societies. We launched this project to assess the understanding of and adherence to international asthma guidelines by clinicians of different specialties, to evaluate possible ameliorative measures, and to identify thresholds at which referral to a respiratory physician or allergist is appropriate. This initiative was supported by both societies and it is our pleasure that two prominent members of the societies' leaderships, namely loana Agache, EAACI President Elect, and Christer Janson, ERS Assembly 6 Secretary, have joined the Task Force as cochairs, offering their expertise and valuable experience. The Task Force includes 21 members and work is already taking place.

\section{Future plans}

In summary, the EAACI-ERS juniors' collaboration has had a very promising and fruitful start. There are already some ideas on how to maintain and further strengthen this relationship in the future. Firstly, we plan to write joint reviews or "landscape" papers to be published in journals of the two societies. The two junior boards will encourage active juniors from both societies to contribute as co-authors. In addition, it is envisaged to provide a platform for joint online educational resources on the topics 
of asthma, allergy and immunology. Last but not least, both committees would like to investigate the possibility to organise a joint research seminar or summer school in the field of asthma, allergy or lung immunology, in order to strengthen our network.

Overall, the initiatives of this collaboration have the possibility to produce significant benefits for both ERS and EAACl, as they will increase the interaction and networking between young researchers working in the field of asthma, allergy and lung immunology. Many of these individuals are among the future clinical and research leaders in their respective fields. This collaboration will facilitate their cooperation in the very best interests of science, medicine and patients with respiratory diseases.

It is the era of international collaborations, and especially the education and career development of young healthcare professionals is of utmost importance for the future of respiratory medicine and research. The ERS-EAACl collaboration is a first, successful step in this direction.

\section{Conflict of interest}

None declared.

\section{References}

1. Skevaki CL, Maggina P, Santos AF, et al. The 10th anniversary of the Junior Members and Affiliates of the European Academy of Allergy and Clinical Immunology. Paediatr Allergy Immunol 2011; 22: 754-757

2. Tomazic PV, Graessel A, Silva AF, et al. A mutually beneficial collaboration between the European Academy of Allergy and Clinical Immunology Junior Members and Clinical and Translational Allergy. Clin Transl Allergy 2016; 6: 43.
3. Demoly P, Adkinson NF, Brockow K, et al. International Consensus on drug allergy. Allergy 2014; 69: 420-437.

4. Papadopoulos NG, Arakawa H, Carlsen K-H, et al. International consensus on (ICON) pediatric asthma. Allergy 2012; 67: 976-997. 